\title{
Irritable bowel syndrome might be associated with dry eye disease
}

\author{
Ioannis Asproudis ${ }^{a}$, Anthoula T. Tsoumania, Konstantinos H. Katsanos ${ }^{b}$, Aristeidis H. Katsanosc, \\ Vasileios Theopistos ${ }^{b}$, Konstantinos A. Paschidis ${ }^{a}$, Epameinondas V. Tsianos ${ }^{b}$, Dimitrios Christodoulou ${ }^{b}$ \\ University of Ioannina, Ioannina, Greece
}

\section{Abstract}

\section{Introduction}

Dry eye disease (DED) or keratoconjunctivitis sicca (KCS) is the most common disease of the ocular surface. In 2007, the International Dry Eye Workshop defined it as "a multifactorial disease of the tears and ocular surface that results in symptoms of discomfort, visual disturbance, and tear film instability with potential damage to the ocular surface. It is accompanied by increased osmolarity of the tear film and inflammation of the ocular surface" [1]. The prevalence of DED is estimated between 7.4 and 33.7\% [2]. Most studies have found an increasing prevalence with aging

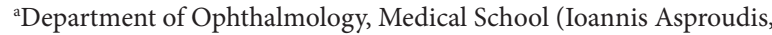
Anthoula T. Tsoumani, Konstantinos A. Paschidis); ${ }^{b} 1^{\text {st }}$ Division of Internal Medicine and Hepato-Gastroenterology Unit (Konstantinos H. Katsanos, Vasileios Theopistos, Epameinondas V. Tsianos, Dimitrios Christodoulou); 'Department of Neurology (Aristeidis H. Katsanos), University of Ioannina Medical School, Ioannina, Greece

\section{Conflict of Interest: None}

Correspondence to: Tsoumani Anthoula, Department of Ophthalmology, Medical School, University of Ioannina, 45110 Ioannina, Greece, Tel.: +30 26510 07528, Fax: +30 2651007025 , e-mail: anthitsoumani@yahoo.gr

Received 3 March 2016; accepted 5 May 2016; published online 16 June 2016

DOI: http://dx.doi.org/10.20524/aog.2016.0064 and a greater prevalence among women probably due to their hormonal status $[2,3]$.

Even though DED is considered as a condition of tear deficiency, either due to decreased lacrimation or excessive evaporation, its pathophysiology seems to be more complex. New concepts of its pathogenesis involve inflammation mechanisms mediated by T-cell lymphocytes $[4,5]$. It has been suggested that it is an inflammatory disorder, which affects both ocular surface and lacrimal glands. Desiccating stress, hyperosmolarity, proinflammatory cytokines released from the lacrimal glands and blinking abnormalities seem to contribute to inflammation of the ocular surface [6-8]. Loss of peptide growth factors and vitamin A, both necessary for cellular proliferation, migration, normal differentiation, immune modulation and ocular wound healing, might also contribute to ocular surface inflammation $[9,10]$.

In some clinical disorders such as Sjögren's syndrome, the lacrimal gland becomes an important target of the immune system resulting in DED. The presence of focal lymphocytic infiltrates and the increased local production of proinflammatory cytokines are characteristic findings of lacrimal gland inflammation [11]. This finding has also been demonstrated by studies investigating the role of anti-inflammatory therapies such as topical $0.005 \%$ cyclosporine, corticosteroid therapy, and tetracycline in patients with DED [12-14].

Irritable bowel syndrome (IBS) is the most common gastrointestinal disorder [15]. It is defined, according to Rome III criteria, as recurrent abdominal pain or discomfort 
for at least 3 days per month during the previous 3 months, associated with two or more of the following symptoms: improvement with defecation, change in the frequency and form or appearance of stools [16]. It affects $10-20 \%$ of adolescents and adults in western societies and is the most common cause of recurrent abdominal pain in children $[17,18]$.

Despite the growing body of literature, IBS pathophysiology remains poorly understood. Recent studies suggest an inflammatory pathogenesis. Several investigators have demonstrated increased numbers of chronic inflammatory cells in the colonic mucosa of patients with IBS [19-21]. T-lymphocytes of the lamina propria are increased both in post-infectious and non-post-infectious IBS patients compared with controls. Mucosal mast cells are also increased, based on quantification methods [22]. Recent documentation of increased numbers of immune cells expressing CD25, a marker of immune cell activation, provides functional evidence of immune activation in IBS [23]. There is also a change in peripheral cytokine profiles in these patients. Abnormal interleukin (IL) -10/-12 ratio has been observed, suggesting a pro-inflammatory state [24].

In our case-controlled study we aimed to investigate the prevalence of DED in IBS compared with healthy subjects who visited our ophthalmology clinic for a regular visual acuity testing.

\section{Patients and methods}

\section{Patients}

The study was conducted at the University Hospital of Ioannina Greece, a referral tertiary center for digestive and ocular diseases. Ninety-five patients with IBS, diagnosed according to Rome III criteria from September 2014 to January 2015 in the Hepato-Gastroenterology Unit, were consecutively included. One experienced gastroenterologist diagnosed all IBS patients before they were further tested for DED. 276 age- and gender-matched individuals without IBS, among the outpatients who had a routine visual acuity testing during the same study period in the Department of Ophthalmology, served as controls. Two ophthalmologists performed all ophthalmic examinations, both of whom were blinded to IBS status.

Patients with pre-existing ocular disease such as glaucoma or uveitis, disorders of the lid or nasolacrimal pathway, previous ocular surgery, and subjects using contact lenses were excluded from the study. We also excluded patients with ocular allergies, pterygium or blepharitis, because these factors may have an influence on dry eye test results (Table 1) [3,25].

For each patient we recorded gender and age. All patients had a routine ophthalmic examination, which included visual acuity measurement, external examination and slit lamp biomicroscopy. All patients were asked to answer a questionnaire regarding dry eye-related ocular symptoms, in the form of oral interview. It comprised 6 questions about itching, sand and burning sensations, red eye appearance, secretions, and discomfort in dry environments [26,27] (Table 2). Each question could be answered with either a "Yes" or a "No". Positive answers to at least three questions of ocular symptoms questionnaire were considered as presence of DED. Additional diagnostic tests for DED, such as Schirmer's test (ST) 1 and tear break-up time (tBUT), were used in this order.

The study was approved from the Institutional Review Board. Informed consent was obtained from all participating patients.

Table 1 Consort flow diagram of patients enrolled

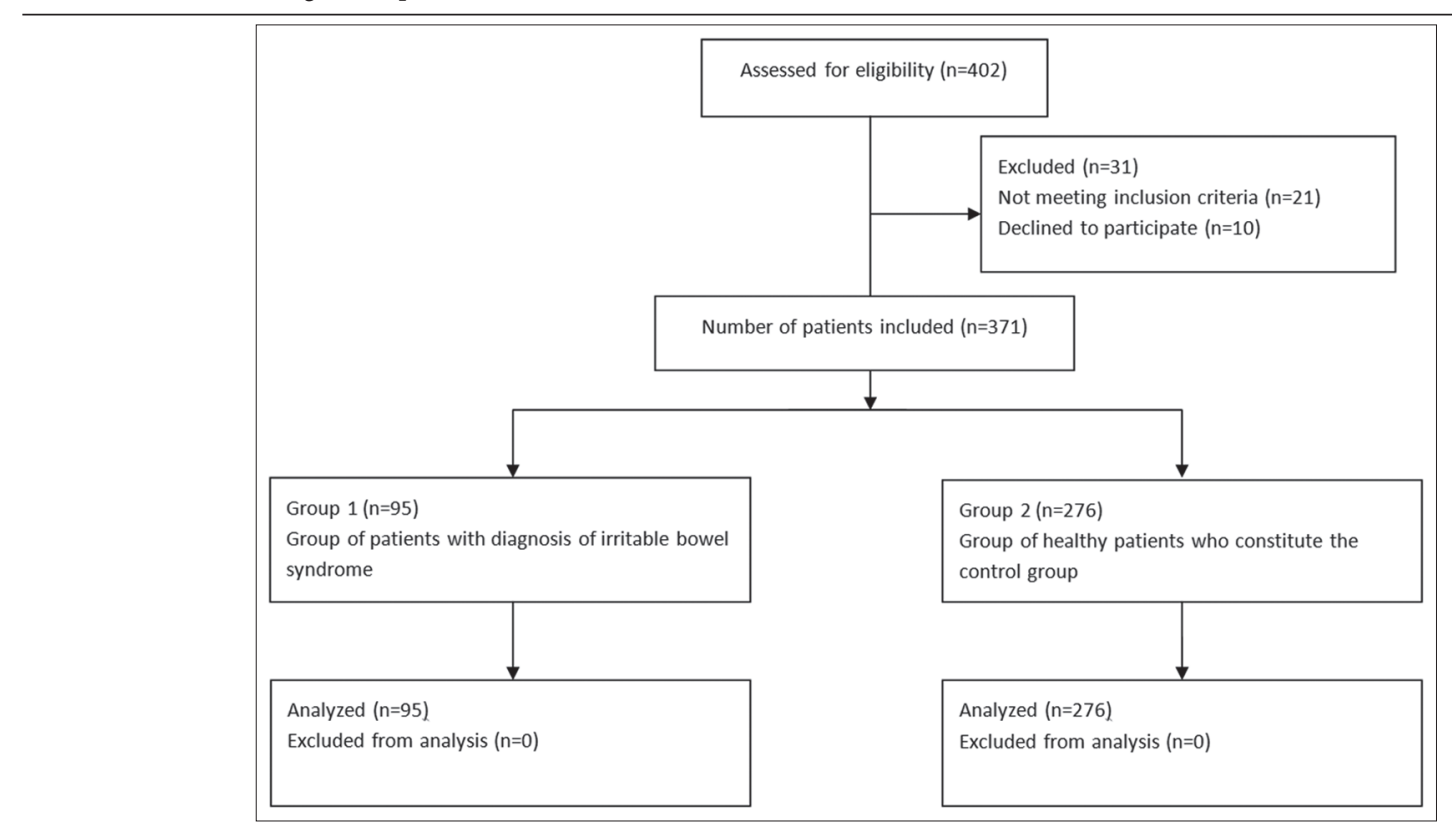




\section{Diagnostic tests}

ST was performed under natural light without topical anesthesia. Standardized filter paper was placed in the lateral canthus away from the cornea and left in place for $5 \mathrm{~min}$, while the eye was closed. The distance moistened was measured in $\mathrm{mm}$. A reading of less than $5 \mathrm{~mm}$ was considered abnormal. It was only performed once in each patient.

Tear film stability was assessed using tBUT via a commercially available fluorescein test paper that came in contact with the bottom conjunctiva. The subjects were instructed to blink 3 times, and then to look straight-forward without any blink. Tear film was observed using a blue cobalt filter under wide lighting. The interval between the last blink and the appearance of the first corneal dry spot was measured. The last procedure was repeated 3 times and the mean value was recorded. A tBUT value less than $10 \mathrm{sec}$ was accepted as abnormal.

\section{Statistical analysis}

We presented data separately for IBS patients and controls. Continuous variables are presented as mean \pm standard deviation (SD, normal distribution), while non-continuous variables are presented as percentages. Normality was assessed with both the graphical inspection of the distribution and with the Shapiro-Wilk normality test. Statistical comparisons between groups were performed using the unpaired t-test or $\mathrm{x}^{2}$ test, as indicated. In case of unequal variances between subgroups the unequal variance assumption was employed, with the use of Welch t-test. Statistical significance for all comparisons was achieved if the $\mathrm{P}$ values from the corresponding statistical tests were below 0.05. All statistical analyses were conducted using the Stata Statistical Software Release 13 for Windows (College Station, TX, Stata Corp LP).

\section{Results}

\section{Patients}

The study group comprised 95 IBS patients and the control group 276 healthy participants. Demographic characteristics for IBS patients and controls are summarized in Table 3. Mean age was $49.8 \pm 15.36$ for women and $58.28 \pm 15.47$ years for men in the IBS group versus $51.9 \pm 16.64$ and $55.8 \pm 19.25$ years respectively in the control group. Both groups matched in gender $(70.5 \%$ females in the IBS group vs. $67.3 \%$ in the control group). However, age difference between the sexes of the same group was greater in IBS patients (8.5 years) than in controls (3.9 years) $(\mathrm{P}=0.280$ for female age, $\mathrm{P}=0.256$ for male age, $t$-test).

\section{Dry eye symptoms in IBS and healthy controls}

More IBS patients gave three or more positive answers than healthy controls (Table 4). Specifically, 68 (72\%) IBS patients
Table 2 Questionnaire of ocular symptoms related to dry eye disease

\begin{tabular}{llll}
\hline $\begin{array}{l}\text { 1. Do you ever feel a foreign body or gritty or } \\
\text { sandy sensation in your eye? }\end{array}$ & Yes & No \\
\hline 2. Do your eyes ever have a burning sensation? & Yes & No \\
\hline 3. Do your eyes ever feel dry? & Yes & No \\
4. Are your eyes ever red? & Yes & No \\
\hline 5. Do you notice much crusting on your lashes? & Yes & No \\
6. Do your eyes ever get stuck shut? & Yes & No \\
\hline
\end{tabular}

Table 3 Comparison of demographic data between patients with irritable bowel syndrome (IBS) and controls

\begin{tabular}{lccc}
\hline & IBS $\mathrm{n}=95$ & Controls $\mathrm{n}=276$ & \\
\hline Female & $67(70.5 \%)$ & $186(67.3 \%)$ & $\mathrm{P}=0.564$ \\
Male & $28(29.5 \%)$ & $90(32.6 \%)$ & $\mathrm{P}=0.576$ \\
Age $(\mathrm{F})( \pm \mathrm{SD}$, years $)$ & $49.8 \pm 15.36$ & $51.9 \pm 16.64$ & $\mathrm{P}=0.280$ \\
Age $(\mathrm{M})( \pm \mathrm{SD}$, years) & $58.28 \pm 15.47$ & $55.8 \pm 19.25$ & $\mathrm{P}=0.256$ \\
\hline F, female; $M$, male; $S D$, standard deviation & & \\
\hline
\end{tabular}

gave at least three positive answers to the questionnaire compared with 116 (42\%) healthy subjects $\left(\mathrm{P}<0.01, \mathrm{x}^{2}\right.$ test $)$.

\section{Tear production in IBS patients compared with healthy control participants}

All clinical tests showed significant differences between groups. Patients with IBS were more likely to have an abnormal ST than healthy controls $(9.26 \mathrm{~mm} / 5 \mathrm{~min} \pm 5.42$ for males with IBS vs. $14.16 \pm 9.09$ for male controls, $\mathrm{P}<0.001$, t-test; and $10.23 \mathrm{~mm} / 5 \mathrm{~min} \pm 6.8$ for females with IBS vs. $13.46 \pm 9.17$ for female controls, $\mathrm{P}=0.002$, t-test). Likewise, IBS patients were more likely to have abnormal tBUT $(7.69 \pm 3.60$ for males and $7.21 \pm 3.15$ for females with IBS vs. $14.02 \pm 6.17$ for male and $14.39 \pm 6.07$ for female controls; both with $\mathrm{P}<0.001$, t-test).

\section{Discussion}

DED is an extremely common disorder that in various grades of severity affects a large part of the general population at one time or another. Millions of people experience symptoms of IBS each year. Both DED and IBS have an unclear pathogenesis, including a common theory of inflammatory pathogenesis.

Over the past few years several studies have shown that dry eye seems to be caused by inflammation mediated by T-cell lymphocytes [5]. A dangerous vicious cycle of inflammation develops on the ocular surface that may lead to ocular surface disease. The role of inflammatory cytokines and matrix metalloproteinases (MMPs) in the pathogenesis of dry eye seems to be very important for both the easier understanding of KCS and for the discovery of new therapeutic agents [7,28]. Increased production and activation of pro-inflammatory cytokines (IL-1, 
Table 4 Schirmer's 1 test, tBUT test, and questionnaire in IBS patients compared with healthy controls

\begin{tabular}{lccc}
\hline & IBS & Controls & P value \\
& Mean values $(\mathrm{n}=95)$ & Mean values $(\mathrm{n}=276)$ & $<.39 \pm 6.07$ \\
\hline tBUT $(\mathrm{F})( \pm \mathrm{SD}, \mathrm{sec})$ & $7.21 \pm 3.15$ & $14.02 \pm 6.17$ & $<0.001$ \\
tBUT $(\mathrm{M})( \pm \mathrm{SD}, \mathrm{sec})$ & $7.69 \pm 3.60$ & $13.46 \mathrm{~mm} / 5 \mathrm{~min}(\mathrm{SD} \pm 9.17)$ & 0.002 \\
Schirmer's test $(\mathrm{F})$ & $10.23 \mathrm{~mm} / 5 \mathrm{~min}(\mathrm{SD} \pm 6.8)$ & $14.16 \mathrm{~mm} / 5 \mathrm{~min}(\mathrm{SD} \pm 9.09)$ & $<0.001$ \\
Schirmer's test $(\mathrm{M})$ & $9.26 \mathrm{~mm} / 5 \mathrm{~min}(\mathrm{SD} \pm 5.42)$ & $116(42)$ & $<0.05$ \\
\hline Questionnaire $\geq 3$ positive answers $(\%)$ & $68(72)$ & &
\end{tabular}

IBS, irritable bowel syndrome; $t B U T$, tear break-up time; $F$, female; $M$, male

IL-6, IL-8, and transforming growth factor- $\beta 1$ ), RNA transcripts, and tumor necrosis factor have been reported in dry eye [29]. MMP-9 appears to play a physiological role in regulating corneal epithelial desquamation. In systemic vitamin A deficiency, there is a reduced expression of MMP-9 and hyperstratification of the corneal epithelium. In contrast, the increased MMP-9 activity in KCS is associated with deranged corneal epithelial barrier function (increased fluorescein permeability), increased corneal epithelial desquamation (punctate epithelial erosions), and corneal surface irregularity $[30,14]$.

As it is widely recognized that inflammation plays a significant role in the etiopathogenesis of dry eye, promoting ocular surface disruption and symptoms of irritation, a number of anti-inflammatory treatments are currently in use for its management. These agents inhibit the expression of inflammatory mediators on the ocular surface, thereby restoring the secretion of a healthy tear film and reducing signs and symptoms. Cyclosporine reduces the inflammation via inhibition of T-cell activation and down-regulation of inflammatory cytokines in the conjunctiva, and lacrimal gland is thus thought to enhance tear production. Topical cyclosporine also increases goblet cell density and decreases epithelial cell apoptosis [31]. Corticosteroids have been reported to decrease the production of a number of inflammatory cytokines [IL$1,-6,-8$, tumor necrosis factor (TNF)- $\alpha$, GM-CSF] and MMP-9 by the corneal epithelium [32]. Tetracyclines also have a variety of anti-inflammatory properties such as inhibition of MMPs and IL-1 production. In ophthalmic practice, doxycyclines have been reported to be effective in dry eye especially induced by acne rosacea and severe blepharitis by reducing irritation symptoms, improving tear film stability, and decreasing the severity of ocular surface disease $[33,34]$.

Similar inflammatory pathogenesis has been reported for IBS. Increased numbers of $\mathrm{T}$ cells in various lymphoid compartments of the small or large intestine in IBS patients have been described. Pro-inflammatory cytokines, such as IL-1 $\beta$, IL-6 and TNF- $\alpha$, in peripheral blood, and mononuclear cells, IL-6, and IL-8 in serum, were reported to be increased in IBS patients. Low-grade inflammation can be detected both through biopsies from intestine and blood testing. Subsequent studies in IBS patients revealed increased numbers of mast cells in the lamina propria of the terminal ileum and mucosa of the colon [35].

To our knowledge, this is the first study investigating the association between IBS and DED based on their inflammatory pathogenesis. Our study indicates that patients with IBS should be considered at increased risk of having DED. ST scores were significantly affected compared with healthy controls. tBUT test also yielded significantly lower results in IBS patients than in controls. In addition, all abnormalities in tear film production resulted in more common ocular complaints of dry eyes (more than 3 positive answers to the dry eye symptoms questionnaire) in IBS patients. Our results are supported by previous studies that demonstrated a relation between IBS and dry eye syndrome. Barton et al in 1999 [36] assessed the frequency of fibromyalgia and sicca complex (dry eyes and mouth) in secondary care patients with IBS. 33\% of 46 IBS patients presented with sicca syndrome compared with only $6 \%$ in the control group, thus adding it to the list of extracolonic manifestations of IBS [36]. In a recent study concerning the prevalence and risk factors of DED in a female cohort, a high association between DED and IBS was found $(\mathrm{P}<0.0005)$. Vehof et al showed that four chronic pain syndromes including pelvic pain, chronic widespread pain, IBS, and dry eye were heritable and shared genetic etiologic factors in common biologic pathways, thus introducing the concept of pain hypersensitivity being a common pathogenetic mechanism $[37,38]$.

Data concerning ocular changes in patients with IBS are limited, and dry eye is often overlooked in the context

\section{Summary Box}

\section{What is already known:}

- Inflammation plays a significant role in the etiopathogenesis of dry eye disease (DED)

- Many studies also suggest an inflammatory pathogenesis for irritable bowel syndrome (IBS)

\section{What the new findings are:}

- We found a possible association between IBS and DED

- A higher prevalence of DED was found in IBS patients than in controls

- DED should be considered when treating IBS patients 
of IBS. Our IBS patients presented a higher prevalence of DED compared with healthy controls. The fact that the score of all tests used for the evaluation of DED was worse in IBS patients than in controls, makes us believe that DED should be considered when treating IBS patients. However, further investigation should be done to establish their pathophysiologic association.

\section{References}

1. The definition and classification of dry eye disease: report of the definition and classification subcommittee of the international dry eye workshop (2007). Ocul Surf 2007;5:75-92.

2. Lin PY, Tsai SY, Cheng CY, Liu JH, Chou P, Hsu WM. Prevalence of dry eye among an elderly Chinese population in Taiwan: The Shihpai eye study. Ophthalmology 2003;110:1096-1101.

3. Moss SE, Klein R, Klein BE. Prevalence of and risk factors for dry eye syndrome. Arch Ophthalmol 2000;118:1264-1268.

4. Gao J, Schwalb TA, Addeo JV, Ghosn CR, Stern ME. The role of apoptosis in the pathogenesis of canine keratoconjunctivitis sicca: the effect of topical cyclosporin A therapy. Cornea 1998;7:654-663.

5. Stern ME, Gao J, Schwalb TA, et al. Conjunctival T-cell subpopulations in Sjogren's and non-Sjygren's patients with dry eye. Invest Ophthalmol Vis Sci 2002;43:2609-2614.

6. Corrales RM, Villarreal A, Farley W, Stern ME, Li DQ, PflugfelderSC. Strain-related cytokine profiles on the murine ocular surface in response to desiccating stress. Cornea 2007;26:579-584.

7. Li DQ, Pflugfelder SC. Matrix metalloproteinases in corneal inflammation. Ocul Surf 2005;3:198-202.

8. Nakamura $S$, Shibuya $M$, Nakashima $H$, et al. Involvement of oxidative stress on corneal epithelial alterations in a blinksuppressed dry eye. Invest Ophthalmol Vis Sci 2007;48:1552-1558.

9. Sommer A. Effects of vitamin A deficiency on the ocular surface. Ophthalmology 1983;90:592-600.

10. Van Setten GB, Viinikka L, Tervo T, Pesonen K, Tarkkanen A, Perheentupa J. Epidermal growth factor is a constant component of normal human tear fluid. Graefes Arch Clin Exp Ophthalmol 1989;227:184-187.

11. Zoukhri D. Effect of inflammation on lacrimal gland function. Exp Eye Res 2006;82:885-898.

12. Sahin A, Bozkurt B, Irkec M. Topical cyclosporine A in the treatment of superior limbic keratoconjunctivitis: a long-term follow-up. Cornea 2008;27:193-195.

13. Hong S, Kim T, Chung SH, Kim EK, Seo KY. Recurrence after topical nonpreserved methylprednisolone therapy for keratoconjunctivitis sicca in Sjögren's syndrome. J Ocul Pharmacol Ther 2007;23:78-82.

14. De Paiva CS, Corrales RM, Villarreal AL, et al. Corticosteroid and doxycycline suppress MMP-9 and inflammatory cytokine expression, MAPK activation in the corneal epithelium in experimental dry eye. Exp Eye Res 2006;83:526-535.

15. Olden KW. Diagnosis of irritable bowel syndrome. Gastroenterology 2002;122:1701-1704.

16. Drossman DA, Dumitrascu DL. Rome III: New standard for functional gastrointestinal disorders. J Gastrointestin Liver Dis 2006;15:237-241.

17. Ringel Y, Drossman DA. Irritable bowel syndrome: classification and conceptualization. J Clin Gastroenterol 2002;35:S7-S10.

18. El-Matary W, Spray C, Sandhu B. Irritable bowel syndrome: the commonest cause of recurrent abdominall pain in children. Eur J Pediatr 2004; 163:584-588.

19. Spiller RC, Jenkins D, Thornley JP, et al. Increased rectal mucosal enteroendocrine cells, $\mathrm{T}$ lymphocytes, and increased gut permeability following acute Campylobacter enteritis and in postdysenteric irritable bowel syndrome. Gut 2000;47:804-811.

20. Chadwick VS, Chen W, Shu D, et al. Activation of the mucosal immune system in irritable bowel syndrome. Gastroenterology 2002;122:1778-1783.

21. Dunlop SP, Jenkins D, Neal KR, Spiller RC. Relative importance of enterochromaffin cell hyperplasia, anxiety, and depression in postinfectious IBS. Gastroenterology 2003;125:1651-1659.

22. Dunlop SP, Jenkins D, Spiller RC. Distinctive clinical, psychological, and histological features of postinfective irritable bowel syndrome. Am J Gastroenterol 2003;98:1578-1583.

23. Collins SM, Barbara G. East meets West: infection, nerves, and mast cells in the irritable bowel syndrome. Gut 2004;53:1068-1069.

24. O'Mahony L, McCarthy J, Kelly P, et al. Lactobacillus and bifidobacterium in irritable bowel syndrome: symptom responses and relationship to cytokine profiles. Gastroenterology 2005;128:541-551.

25. Lee AJ, Lee J, Saw SM, et al. Prevalence and risk factors associated with dry eye symptoms: a population based study in Indonesia. $\mathrm{Br}$ J Ophthalmol 2002;86:1347-1351.

26. Bandeen-Roche K, Muñoz B, Tielsch JM, West SK, Schein OD. Self-reported assessment of dry eye in a population based setting. Invest Ophthalmol Vis Sci 1997;38:2469-2475.

27. Schein OD, Tielsch JM, Munõz B, Bandeen-Roche K, West S. Relation between signs and symptoms of dry eye in the elderly-a population-based perspective. Ophthalmology 1997;104:1395-1401.

28. Gumus K, Cavanagh DH. The role of inflammation and antiinflammation therapies in keratoconjunctivitis sicca. Clin Ophthalmol 2009;3:57-67.

29. Niederkorn JY, Stern ME, Pflugfelder SC, et al. Desiccating stress induces T cell-mediated Sjögren's Syndrome-like lacrimal keratoconjunctivitis. J Immunol 2006;176:3950-3957.

30. Luo L, Li DQ, Doshi A, Farley W, Corrales RM, Pflugfelder SC. Experimental dry eye stimulates production of inflammatory cytokines and MMP-9 and activates MAPK signaling pathways on the ocular surface. Invest Ophthalmol Vis Sci 2004;45:4293-4301.

31. Hessen M, Akpek EK. Dry eye: an inflammatory ocular disease. J Ophthalmic Vis Res 2014;9:240-250.

32. Djalilian AR, Nagineni CN, Mahesh SP, Smith JA, Nussenblatt RB, Hooks JJ. Inhibition of inflammatory cytokine production in human corneal cells by dexamethasone, but not cyclosporine. Cornea 2006;25:709-714.

33. Akpek EK, Merchant A, Pinar V, Foster CS. Ocular rosacea: patient characteristics and follow-up. Ophthalmology 1997;104:1863-1867.

34. Gilbard JP. Dry eye, blepharitis and chronic eye irritation: divide and conquer. J Ophthalmic Nurs Technol 1999;18:109-115.

35. Akiho H, Ihara E, Nakamura K. Low-grade inflammation plays a pivotal role in gastrointestinal dysfunction in irritable bowel syndrome. World J Gastrointest Pathophysiol 2010;15;97-105.

36. Barton A, Pal B, Whorwell PJ, Marshall D. Increased prevalence of sicca complex and fibromyalgia in patients with irritable bowel syndrome. Am J Gastroenterol 1999;94:1898-1901.

37. Vehof J, Kozareva D, Hysi PG, Hammond CJ. Prevalence and risk factors of dry eye disease in a British female cohort. Br J Ophthalmol 2014;98:1712-1717.

38. Vehof J, Zavos HM, Lachance G, Hammond CJ, Williams FM. Shared genetic factors underlie chronic pain syndromes. Pain 2014;155:1562-1568. 